\title{
Development of a 3D Method Using SEM and Liquid-Phase STEM to Study Cell Morphology
}

Akkiz Bekel $^{1 *}$, Louis-Marie Lebas ${ }^{1}$, Lucian Roiban $^{1}$, Cyril Langlois ${ }^{1}$, Mayra Yucely Beb $\mathrm{Caal}^{2}$, Sounkalo Dembélé ${ }^{2}$, Nadine Piat ${ }^{2}$, Claire Gaillard ${ }^{1}$, Bérangère Lesaint ${ }^{1}$, and Karine MasenelliVarlot $^{1}$

${ }^{1}$ Univ Lyon, INSA Lyon, UCBL, CNRS, MATEIS, UMR5510, 69621 Villeurbanne, France.

${ }^{2}$ FEMTO-ST Institute, Univ. Bourgogne Franche-Comté, CNRS, UFC, ENSMM, 15B avenue des Montboucons, 25030 Besançon cedex, France.

Cell adhesion is involved in many cellular processes such as proliferation, migration and differentiation. Cell adhesion conditions cell morphology, indeed recent studies have shown that the substrate on which cells are cultured can lead to different cell morphologies [1]. A challenging investigation related cell morphology and gene expression in response to nanotopography [2]. The morphological analysis is mostly done in 2D using high vacuum scanning electron microscopy (HVSEM). We aim to set a 3D analysis method using HVSEM and liquid-phase SEM (LPSEM) to reduce changes occurring during sample preparation, such as dehydration. A home-made scanning transmission electron microscopy (STEM) tomography device, specific to LPSEM, has previously been developed to study aqueous suspensions in 3D [3].

\section{HVSEM 3D}

3T3 mouse embryonic fibroblasts were seeded onto titanium, 24 hour later cells were fixed and dehydrated. Images were recorded at different tilt and rotation angles by collecting secondary electrons using a Thermofischer QuattroS Environmental SEM (ESEM) operated in HV mode at $15 \mathrm{keV}$. The 3D object was obtained using Meshroom, a 3D reconstruction software (Figure 1), or a lab-made software in development, pollen3d (not shown).

\section{LPSEM-STEM}

3T3 mouse embryonic fibroblasts were seeded onto a gold TEM grid covered with a fibronectin coated carbon film, then 24-hour cells were fixed and kept in buffer. Images were acquired by recording Bright Field (BF) and High Angle Annular Dark Field (HAADF) detectors at different tilt angles (Figure 2A and B). The tilt images were aligned using the software Imod, the volumes were computed using the TomoJ plugin of ImageJ. Data segmentation and the 3D model were performed using ImageJ and 3D Slicer.

HVSEM and LPSEM-STEM enable a 3D analysis of whole cells with complementary morphological information such as cell spreading and filopodia formation. The limits of the techniques will be explored in terms of cell thickness, spatial resolution and electron dose received by the sample. In particular, we show that the overall cell morphology can be reconstructed using HVSEM 3D, although some noise is introduced into the reconstructed surface. Successful reconstructions of a $3-\mu \mathrm{m}$ thick cell are also obtained using BF and HAADF tilt series in LPSEM-STEM. However, the cell thickness should not exceed a maximum value for HAADF tilt series. The comparison between both segmented models (obtained from $\mathrm{BF}$ and HAADF series) indicate an uncertainty of $13 \pm 6 \%$ on the cell volume. Such uncertainty is attributed to the difficult segmentation between the cell outline and the artifacts due to the missing wedge, especially in HAADF when the gold particles are not visible on the cell. A solution could come from the 
combination of both 3D volume and surface reconstruction procedures. Such development opens the way towards 3D analysis of intact cells in environments mimicking their native environment.

[1] C-Y Yang et al, European Cells and Materials 20 (2010), p. 415.

[2] MFA Cutiongco et al, Nature Communications 11 (2020), p. 1.

[3] K Masenelli-Varlot et al, Microscopy and Microanalysis 20 (2014), p. 366.

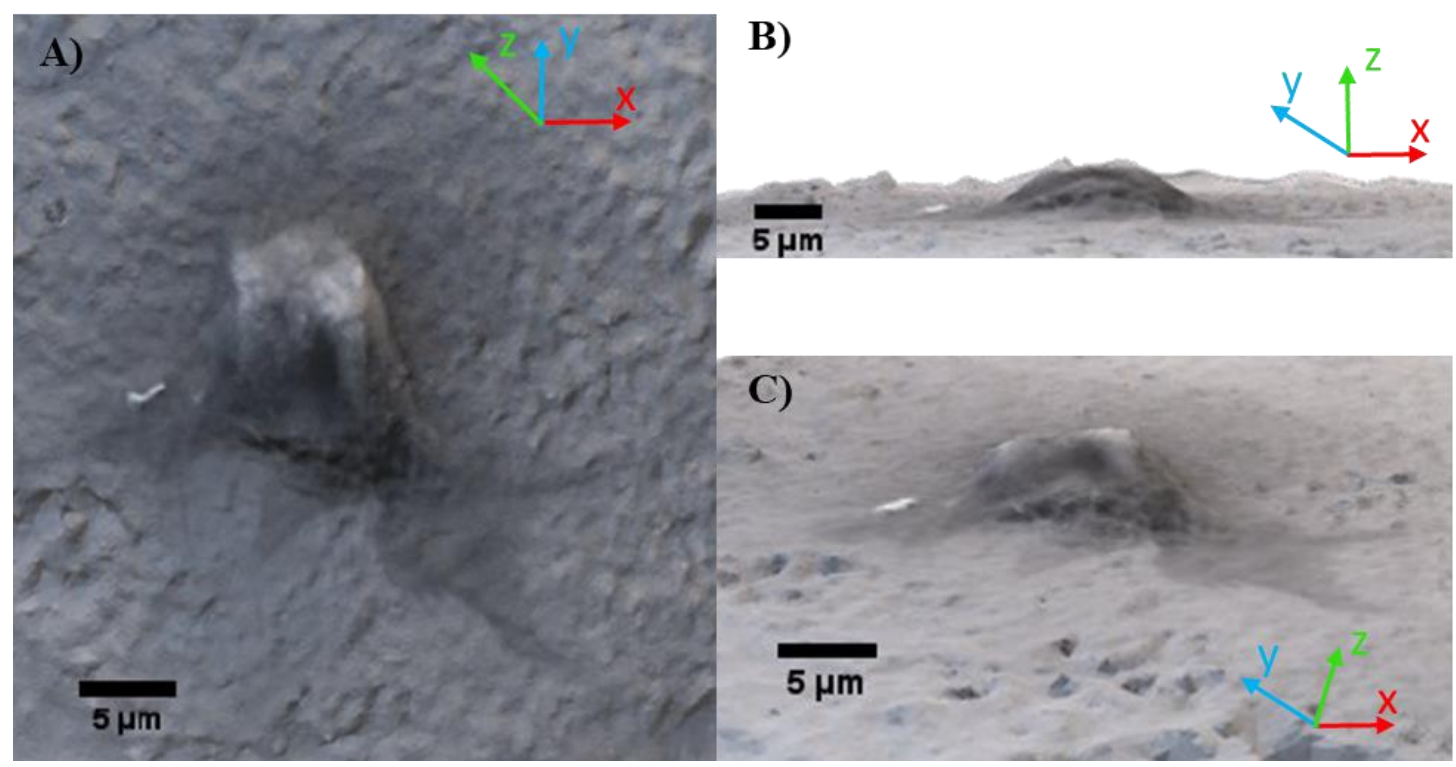

Figure 1. Top view (A), side view (B) and oblique view (C) of a reconstructed embryonic mouse fibroblast cell volume cultivated onto titanium from secondary electron images collected at different tilt angles.
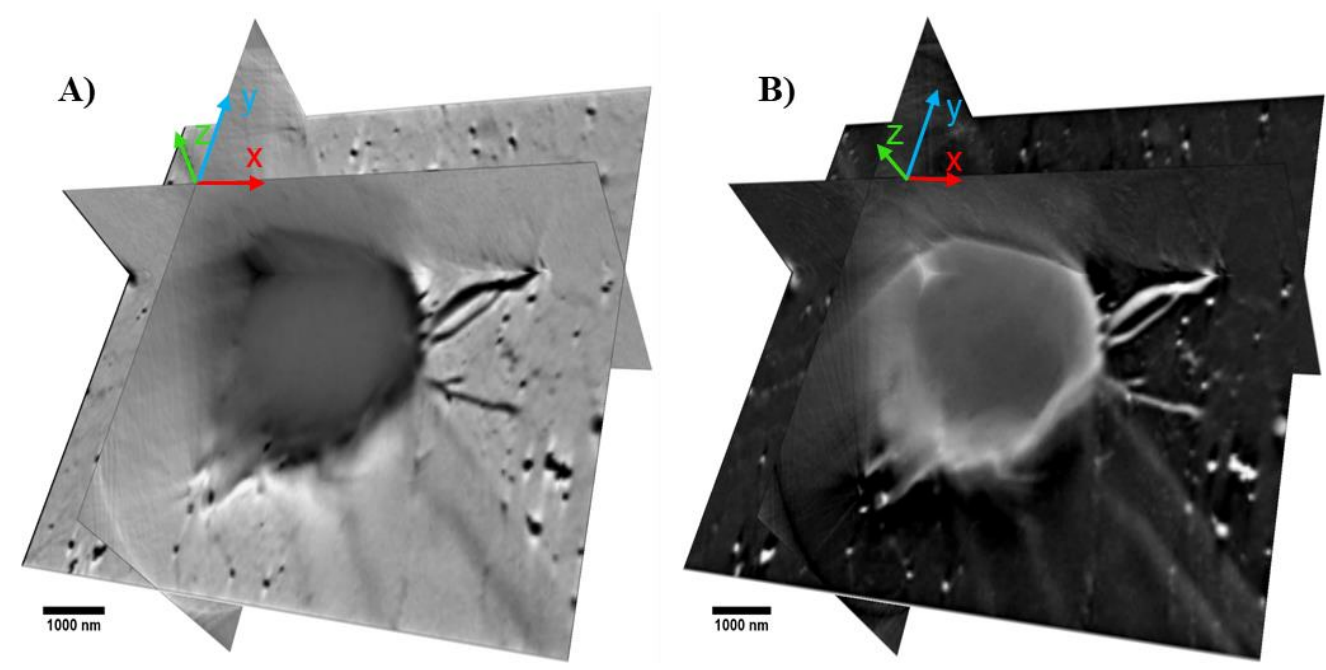

Figure 2. Orthogonal cross-sections of the reconstructed embryonic mouse fibroblast cell volume from images collected at different tilt angles in BF A) or HAADF B) mode. 